\title{
Proteomic analyses of monocytes obtained from Hispanic women with HIV-associated dementia show depressed antioxidants
}

\author{
Stephanie Kraft-Terry ${ }^{1}$, Yamil Gerena ${ }^{2,3}$, Valerie Wojna ${ }^{2,4}$, Marines Plaud-Valentin ${ }^{2}$, \\ Yolanda Rodriguez ${ }^{2}$, Pawel Ciborowski ${ }^{1}$, Raul Mayo ${ }^{2,5}$, Richard Skolasky ${ }^{6}$, Howard E. \\ Gendelman $^{1}$, and Loyda M. Meléndez ${ }^{2,7}$ \\ ${ }^{1}$ Department of Pharmacology and Experimental Neuroscience, University of Nebraska Medical \\ Center, Omaha, NE \\ 2 Specialized Neuroscience Program in NeuroAIDS, University of Puerto Rico Medical Sciences \\ Campus, San Juan, PR \\ 3 School of Pharmacy, University of Puerto Rico Medical Sciences Campus, San Juan, PR \\ ${ }^{4}$ Department of Internal Medicine, Neurology Division, University of Puerto Rico Medical \\ Sciences Campus, San Juan, PR \\ ${ }^{5}$ Department of Physical Medicine, University of Puerto Rico Medical Sciences Campus, San \\ Juan, PR \\ ${ }^{6}$ Department of Orthopedic Surgery, Johns Hopkins University, Baltimore, MD \\ 7 Department of Microbiology, School of Medicine, University of Puerto Rico Medical Sciences \\ Campus, San Juan, PR
}

\section{Abstract}

Monocyte ingress into the brain during progressive human immunodeficiency virus (HIV-1) infection parallels the severity of cognitive impairments. Although activated monocyte phenotypes emerge during disease, the functional correlates of these cells remain unresolved. To this end, we studied the proteome of blood-derived monocytes obtained from Hispanic women with the most severe form of HIV-associated neurocognitive disorder, HIV-associated dementia (HAD). Monocytes isolated from peripheral blood mononuclear cells by CD14+ immunoaffinity column chromatography were $>95 \%$ pure. Cells were recovered from five patients without evidence of cognitive impairment and four with HAD and analyzed by two-dimensional difference gel electrophoresis and tandem mass spectrometry. Importantly, ADP ribosylhydrolase, myeloperoxidase, thioredoxin, peroxiredoxin 3, NADPH, and GTPase activating protein were all downregulated in HAD. In regards to myeloperoxidase, thioredoxin, and peroxiredoxin 3 these changes were validated in an additional cohort of 30 patients by flow cytometry. We conclude that deficits in monocyte antioxidant proteins lead to neuronal damage through the loss of hydrogen peroxide scavenging capabilities, thus exposing neurons to apoptosis-inducing factors. Altered monocyte functions therefore may contribute to the development and progression of HAD.

\section{Keywords}

monocytes; proteomics; 2D-DIGE; mass spectrometry; HIV-1; HAND; HAD

\footnotetext{
*Corresponding Author: Loyda M. Meléndez, PhD, Professor, Department of Microbiology, School of Medicine, Medical Sciences Campus, San Juan, Puerto Rico 00935, Phone: 787-758-6132, Fax: 787-777-0078, loyda.melendez@upr.edu.
} 


\section{Statement of Clinical Relevance}

HIV-associated neurocognitive disorder (HAND) affects up to $50 \%$ of virus-infected people during the course of disease. Clinical features of neurocognitive dysfunction ranges from asymptomatic neurocognitive impairment, to minor cognitive motor disorder, to severe cognitive, motor, and behavioral impairments. The latter is called HIV-associated dementia (HAD) and can affect up to 7\% of infected people. Despite the advent of antiretroviral therapy (ART), the incidence of HAND has remained constant. However, the diagnosis of HAND is made after a battery of neuropsychological tests and after exclusion of opportunistic infections, psychiatric disorders, and malignancies. Early biomarkers are desperately needed to detect, stage, and monitor disease when therapeutic interventions would prove most helpful. Because HIV-1-infected monocytes pass through the blood-brain barrier and differentiate to perivascular macrophages, we chose to analyze their potential proteomic alterations in a well-characterized Hispanic longitudinal cohort of HIV-1-infected ART-treated women. A significant decrease in specific antioxidant proteins during progressive cognitive impairment were discovered and validated by flow cytometry testing. These studies support the significance of the role of circulating monocytes in the neuropathogenesis of HIV-1 infection and provide novel blood markers that can readily be applied to the clinic.

\section{Introduction}

HIV-associated neurocognitive disorder (HAND) affects up to 50\% of HIV-infected people during the course of viral infection. Disease severity for HAND ranges from asymptomatic neurocognitive impairment, to minor cognitive motor disorder, to severe cognitive, motor, and behavioral impairments or HIV-associated dementia (HAD) [1]. HAD can affect 7\% of infected people during the disease course. Despite the advent of antiretroviral therapy (ART), the incidence of HAND has remained constant albeit in less debilitating forms [2]. The underlying mechanisms for neuronal dysfunction or death in disease involve neuroinflammatory responses heralded by infiltrating HIV-1-infected mononuclear phagocytes (MPs; blood-borne monocytes and macrophages and microglia) [3]. Infiltrating MPs carry infection from the periphery to the central nervous system (CNS) and subsequently initiate a cascade of glial (astrocytes and microglia) autocrine and paracrine immune events, resulting in the secretion of pro-inflammatory neurotoxic factors and viral proteins [4-6]. MPs also produce chemokines that attract further inflammatory cell CNS infiltration that perpetuates the neurotoxic cascade [7,8]. Once inside the brain, HIV-1infected MPs establish a long-lived viral reservoir and serve as a continuous source of neurotoxins [9].

MPs are responsible for scavenging cellular debris, regulating oxidative stress, and protecting the host from outside insults. Lack of control of oxidative stress may also contribute to neurodegeneration during progressive viral infection. Nonetheless, alterations, if any, that occur in peripheral monocytes that influence their destructive effects as they ingress within the CNS are not known. What is known is that homeostatic maintenance functions of blood-borne monocytes are broken and parallel the metabolic changes leading to an encephalopathy. These metabolic changes are characterized by behavioral, motor, and cognitive impairments [10].

In recent years proteomic analyses of body fluids [plasma and cerebrospinal fluid (CSF)] and monocytes from HIV-1-infected individuals with cognitive impairment have uncovered protein changes that reflect CNS disease [11-13]. Parallel studies investigated HIV-1- 
infected monocyte-derived macrophages (MDM) [14-16], yielding a broad range of proteins linked to cytoskeleton remodeling, motility, oxidative regulation, vesicular trafficking, and immune responses. Because HIV-1-infected monocytes pass through the blood-brain barrier and differentiate to perivascular macrophages, we chose to analyze the changes induced in monocytes from patients with HAD in a well-characterized Hispanic longitudinal cohort of HIV-1-infected ART-treated women. We found a significant decrease in specific antioxidant proteins during progressive cognitive impairment. These studies support the significance of the role of circulating monocytes in the neuropathogenesis of HIV-1 infection and provide new therapeutic targets for disease.

\section{Materials and Methods}

\section{Patients}

The Hispanic/Latino Longitudinal NeuroAIDS cohort of HIV-seropositive women with or without cognitive dysfunction was recruited from two well-established primary HIV clinics in Puerto Rico [17]: the Latin American Center for Sexually Transmitted Diseases at the Puerto Rico Medical Center and the Center of Maternal-Infant HIV Infections at the University of Puerto Rico Medical Sciences Campus (UPR-MSC). The selection criteria were HIV-1-infected Puerto Rican women at least 18 years of age, with CD4+ T lymphocyte counts of $\leq 500$, at least a $9^{\text {th }}$ grade education, and no evidence of either active systemic infection or neurodegenerative disease. The educational level was a requirement for the neuropsychological tests. The clinical study was approved by the UPR-MSC Institutional Review Board (IRB) and was conducted with the informed consent of the participants.

\section{Evaluations of participants}

Evaluations were performed as described previously in Wojna et al., 2006. Following consent, individual participants were required to provide demographics and pertinent medical histories. The information included age at enrollment and the most likely mode of HIV-1 transmission. Evaluation of cognitive function included history and neuropsychological tests. Plasma and CSF viral loads were determined using Ultrasensitive RNA Roche Amplicor determination, at an AIDS Clinical Trial Group (ACTG) Certified Laboratory, of CD4+ and CD8+ T-cell counts by flow cytometry. Lumbar punctures were performed once a year. Neurological evaluation consisted of a mental status examination, sensory functions (including response slowing, speed of thought, and language), testing of behavior and mood, as well as standard neurologic evaluations of cranial nerves, cerebellar, motor, reflexes, and sensory evaluations. The neuropsychological evaluation included the tests described previously $[1,17]$. These included tests of verbal memory (subtests $5^{\text {th }}$ trial, memory, and recognition of the Rey Auditory Verbal Learning Test), frontal executive function (Stroop word/color and Trail Making B), psychomotor speed (Symbol Digit Modalities Test and visual and auditory reaction time non dominant hand), and motor speed (Trail Making A and Grooved Pegboard dominant and nondominant hand). All tests were conducted on all patients in Spanish. The Beck Depression Index was used to screen for depression. Both the neurologist and the neuropsychologist were blinded to each other's findings. To develop normative values (z-scores) of the neuropsychological tests in Puerto Rican women, we identified a group of HIV-1-seronegative women matched for age, education, and sociodemographic status.

Cognitive impairment was determined with the use of the American Academy of Neurology HIV-associated dementia criteria [1]. In this study 39 HIV-1-seropositive women characterized for cognitive function were accrued ( 20 with normal cognition and 19 with HAD). 


\section{Monocyte isolation from the HIV-infected women cohort}

CD14+ monocytes were isolated from peripheral blood mononuclear cells (PBMC) of HIVseropositive patients by immune chromatography columns containing antibodies against CD14 (Miltenyi Biotech) with high purity (98\% CD14+) and protein recovery.

\section{Sample purification and preparation}

Cell lysates were prepared from CD14+ purified monocytes from five HAD and five normal control donors. These lysates were purified using 2D Clean-up (GE Healthcare) and resuspended in $100 \mu \mathrm{L} 0.1 \%$ TritonX-100 (Fisher). Acquired protein was quantified using a Biorad Dc protein quantification kit. Five micrograms of protein was aliquoted from each sample, and another $5 \mu \mathrm{g}$ from each sample was pooled for an internal standard. Samples were dried by speedvac and resuspended in DIGE lysis buffer (30 mM Tris pH 8.0, $7 \mathrm{M}$ urea, $2 \mathrm{M}$ thiourea, $4 \% \mathrm{CHAPS}$ ). Utilizing saturation labeling for low abundant samples (GE Healthcare), these were reduced by treatment with $2 \mathrm{nmol} \mathrm{TCEP}$ for 1 hour at $37^{\circ} \mathrm{C}$ and labeled with $4 \mathrm{nmol} \mathrm{Cy} 5$ for experimental samples and $\mathrm{Cy} 3$ for the pooled internal standard for 30 minutes at $37^{\circ} \mathrm{C}$. The reaction was stopped with the addition of $2 \mathrm{x}$ sample buffer (7 M urea, $2 \mathrm{M}$ thiourea, 4\% CHAPS, 2\% pharmalyte, $130 \mathrm{mM}$ DTT) [11].

PBMCs were obtained from leukophoresis at the University of Nebraska Medical Center. They were labeled with CD14+ selection micro beads (Miltenyi Biotech) and passed through an MS positive selection column (Miltenyi Biotech) for magnetic separation. Monocyte purity was examined by staining with CD14-FITC antibody (Miltenyi Biotech) and analyzed using FACS. Purity was determined to be $>99 \%$. Monocytes were lysed using DIGE lysis buffer, purified using a 2D-Clean-up kit, and quantified using a 2D-Quant kit (GE Healthcare). Four hundred micrograms of protein was reduced with $200 \mathrm{nmol}$ TCEP for 1 hour at $37^{\circ} \mathrm{C}$ followed by labeling with $400 \mathrm{nmol} \mathrm{Cy} 3$ for 30 minutes at $37^{\circ} \mathrm{C}$. Reaction was stopped by adding $1 \mathrm{x}$ sample buffer ( $7 \mathrm{M}$ urea, $2 \mathrm{M}$ thiourea, $4 \%$ CHAPS) to a total volume of $445.5 \mu \mathrm{L}, 4.5 \mu \mathrm{L}$ pharmalytes, and $4.5 \mathrm{mg}$ of DTT.

\section{Isoelectric focusing}

In-gel rehydration was performed combining equal volumes of labeled sample and internal standard for analytical gels or Cy3 labeled monocyte lysates for preparative gel electrophoresis with rehydration buffer (7 M urea, $2 \mathrm{M}$ thiourea, 4\% CHAPS, $1 \%$ pharmalytes, $13 \mathrm{mM}$ DTT). Samples were then loaded into the rehydration chamber with a pH 3-10, 24-cm immobiline dry strip (GE Healthcare), covered with dry strip cover fluid (GE Healthcare), and incubated at room temperature overnight. Rehydrated strips were transferred to Ettan IPGphor II for $1^{\text {st }}$ dimension separation (isoelectric focusing, IEF). IEF was carried out at a constant $20^{\circ} \mathrm{C}$ for $93.7 \mathrm{kVh}(500 \mathrm{~V}$ for $0.5 \mathrm{kVh}$, gradient to $1000 \mathrm{~V}$ for $6 \mathrm{kVh}$, gradient to $8000 \mathrm{~V}$ for $13.5 \mathrm{kVh}, 8000 \mathrm{~V}$ for $30 \mathrm{kVh}$, gradient to $10000 \mathrm{~V}$ for 16.5 $\mathrm{kVh}, 10000 \mathrm{~V}$ for $27.2 \mathrm{kVh}$ ) [11].

\section{D difference gel electrophoresis (2D-DIGE)}

After IEF, strips were incubated in equilibration solution (6 M urea, $0.1 \mathrm{M}$ Tris $\mathrm{pH} 8.0,30 \%$ glycerol, $2 \%$ SDS, $0.5 \%$ DTT) for 15 minutes with gentle agitation. Strips were then loaded onto the top of 10-20\% gradient polyacrylamide gels and fixed with $0.5 \%$ agarose sealing solution containing bromophenol blue. Second dimension separation was carried out using the Ettan Daltsix Electrophoresis System (GE Healthcare) at $25^{\circ}$ C. Current was held constant at $10 \mathrm{mAmps}$ per gel for the first hour and then at $12 \mathrm{mAmps}$ per gel overnight, until bromophenol blue reached the gel bottom. Visualization of gel spots was performed using a Typhoon 9410 Variable Mode Imager (GE Healthcare, Piscataway, NJ). Gels were scanned at $100 \mu \mathrm{m}$ resolution collecting signals for $\mathrm{Cy} 3$ and $\mathrm{Cy} 5$ at excitation wavelengths 
of 520 and $620 \mathrm{~nm}$, respectively. Images were imported into DeCyder 6.5 (GE Healthcare, Piscataway, NJ) software for spot detection and analysis of differential expression through biological variation analysis. One gel from a HAD sample was not used for analysis due to poor protein separation leaving four HAD samples for analysis. Spots with p-values $\leq 0.05$ were selected for spot picking by means of an automatic Ettan Spot Picker (GE Healthcare, Piscataway, NJ) using a 2.0-mm diameter picking head [11].

\section{In-gel tryptic digestion}

Gel pieces were washed with $200 \mu \mathrm{L} 50 \% \mathrm{H}_{2} \mathrm{O} / 50 \%$ acetonitrile (ACN) for 5 minutes, 200 $\mu \mathrm{L} 50 \% \mathrm{ACN} / 50 \mathrm{mM} \mathrm{NH}_{4} \mathrm{HCO}_{3}$ for 30 minutes, $200 \mu \mathrm{L} 50 \% \mathrm{ACN} / 10 \mathrm{mM} \mathrm{NH}_{4} \mathrm{HCO}_{3}$ for 30 minutes. Gel pieces were dried by speedvac, treated with trypsin (Promega) and 50 $\mu \mathrm{L} 10$ $\mathrm{mM} \mathrm{NH} \mathrm{HCO}_{3}$, and incubated overnight at $37^{\circ} \mathrm{C}$. Peptides were extracted through incubation of spots with $200 \mu \mathrm{L} 0.1 \%$ TFA, $60 \%$ ACN for 2 hours. Extracted peptides were dried using a speedvac concentrator. Peptides were resuspended in $20 \mu \mathrm{L} 0.5 \%$ TFA and purified using ZipTips (Millipore, Billerica, CA) following the manufacturer's protocol.

\section{Protein identification}

Peptides were fractionated on a microcapillary RP-C18 column (NewObjectives, Woburn, MA) followed by fragmentation using the ESI LC MS/MS system (ProteomeX System with LCQDecaPlus, ThermoElectron, Inc., San Jose, CA) in a nanospray configuration. The spectra obtained from mass spectrometric analyses were searched with the use of the Sequest search engine (BioWorks 3.2 software from ThermoElectron Inc., San Jose, CA). For high-confidence protein identification, at least two unique peptides sequenced were required [11].

\section{Flow cytometry}

Two-color flow cytometry was used for intracellular detection of antioxidant expression in monocytes from frozen PBMC of HIV-positive women. Fifteen samples of each group (normal cognition and HAD) were tested independently for intracellular expression of antioxidants. PBMC were thawed and incubated with CD14-PE monoclonal antibody (BD Biosciences, CA) for 30 minutes at $4 \mathrm{oC}$. The cells were washed two times with PBS (1X)/ FBS (3\%) by centrifugation at 1,100 rpm for 5 minutes at $40 \mathrm{C}$. Cells were permeabilized with cytoperm/cytofix solution (BD Biosciences, CA) for 20 minutes followed by incubation with FITC conjugated antibodies against SOD-1, peroxiredoxin, and myeloperoxidase, thioredoxin for 30 minutes, washed by centrifugation and fixed with $0.5 \%$ paraformaldehyde. Cells were analyzed by flow cytometry in a FACS Calibur (BD Biosciences). The Cell Quest software (BD Biosciences, CA) was used for data acquisition and multivariate analysis. Monocytes were gated in FSC vs. SSC dot plot by size and granularity, and subsequently the CD14+ cells were identified in FL2 dot plot. Intracellular markers were identified in the FL1 channel. Ten thousand events were evaluated for each sample and the mean fluorescence intensity (MFI) of the cells was determined from the median peak channel of the histograms. Data on scatter parameters and histograms were acquired in $\log$ mode. Monocyte autofluorescence was subtracted from the fluorescence intensity values of the stained samples.

\section{Statistical methods}

Hotelling's t-squared statistic was used to significance for flow cytometry data $(\mathrm{p} \leq 0.05)$. Hotelliing's t-squared statistic is analogous to Student's t-test in that it makes comparisons between two groups. It differs in that it simultaneously adjusts for multiple comparisons, thereby preserving overall experimental Type I error. One-way ANOVA analysis was used to evaluate significance for 2D-DIGE data $(\mathrm{p} \leq 0.05)$. 


\section{Results}

\section{Participants}

Table 1 lists the demographic, neurologic, immune, and viral statuses of the HIV-1seropositive Hispanic women included in this study. Those with HAD were older on average than those with normal cognition (44 years vs. 38 years, $\mathrm{p}=.015$ ). There was no difference between those with normal cognition and those with HAD with respect to CD4 or CD8 cell counts, plasma HIV viral load, or type of anti-retroviral therapy use. For this study, individuals with no histories of intravenous drug abuse or co-infections were selected.

\section{D-DIGE and protein identification by LC MS MS}

2D-DIGE analysis identified a between 4221 and 4506 spots in each individual gel. After matching all 9 gels together 18 spots were determined to be significantly differentially regulated between normal cognition and HAD with $\mathrm{p} \leq 0.05$. Six of these significant spots had a fold change $\geq 1.5$ or $\leq-1.5$, the arbitrary cut-off value that was selected (Table 2 ). Because the fold changes observed in this experiment were modest, we chose a slightly less stringent cut-off with the understanding that we must validate our findings [18]. Three of these downregulated spots were identified to be anti-oxidant proteins: myeloperoxidase, peroxiredoxin 3, and thioredoxin (Figure 1). Because we previously identified differences in antioxidant protein levels in individuals with HAD [19], we chose these proteins to be validated by flow cytometry from frozen PBMC of an additional 30 participants from the cohort.

\section{Validation by flow cytometry}

Following membrane permeabilization of the CD14+ monocytes and immunolabelling with antibodies against cytoplasmic proteins identified by 2D-DIGE, a statistically significant decrease between those with normal cognition and those with HAD was seen $(p \leq 0.05)$ for peroxiredoxin and thioredoxin. There was no statistical difference in myeloperoxidase expression between those with normal cognition and those with HAD. This finding was most likely a false positive identified as a result of our lower threshold for fold-change values. We also confirmed decreased levels of $\mathrm{Cu} / \mathrm{Zn}$ superoxide dysmuthase (SOD) in peripheral blood monocytes by flow cytometry. SOD was not identified downregulated by 2D DIGE. Biological variability may have resulted in masking the identification of changes in SOD due to a small sample size. Proteins can also co-migrate to the same location as SOD on the 2D gel. If the protein has the opposite expression of SOD it can mask the change in SOD and therefore we would not identify it with 2D DIGE. SOD is an antioxidant protein we identified previously to be downregulated in CD14+ monocytes of HADaffected individuals by ELISA [19]. No significant difference was seen in the expression of myeloperoxidase as demonstrated by decreased MFI and a shift to the left in the histograms from normal cognition and HAD patients (Figure 2).

We examined each marker independently, and, after adjusting for multiple comparisons using Hotelling's t-squared, found significant down-regulation in monocytes expressing CD14/SOD ( $\mathrm{p}=.032)$, CD14/thioredoxin ( $\mathrm{p}=.006)$, and CD14/peroxiredoxin ( $\mathrm{p}=.012)$.

\section{Discussion}

These studies were performed to identify biomarkers that could be linked to cognitive impairments in HIV-1-infected women. Previous data on other HIV NeuroAIDS cohorts have been predominately from male participants. A longitudinal NeuroAIDS cohort with antiviral therapy was established in 2002 as part of a Specialized Neuroscience Research Program, at the University of Puerto Rico. This cohort is well characterized [17]. 2D-DIGE 
analysis of monocyte samples from this cohort provided six possible protein targets that appeared to be differentially regulated between normal cognition and HAD. All six of these proteins were decreased in HAD. Although differences in age were found between those with normal cognition and those with HAD, they did not correlate with the expression of antioxidants (data not shown).

We chose to validate thioredoxin, peroxiredoxin, and myeloperoxidase because of their role in the control of oxidative stress. Thioredoxin and peroxiredoxin were validated by flow cytometry, but myeloperoxidase did not differ between patients with and those without HAD. The downregulation of both thioredoxin and peroxiredoxin in peripheral monocytes implies decreased antioxidant capabilities of peripheral monocytes in patients with HAD. Because we previously demonstrated a downregulation in SOD-1 in monocytes from women with HAD by ELISA, we wanted to validate this finding by flow cytometry as well, as viable cells from the longitudinal cohort and this method do not require physical separation of cells which is best for analysis of different cell populations in a small sample size. We obtained comparable results, further confirming that peripheral monocytes have decreased antioxidant proteins in individuals with HAD.

Thioredoxin is an antioxidant protein that facilitates the reduction of proteins by cysteine thiol-disulfide exchange through two redox-active cysteine residues in its active center. Thioredoxin operates with NADPH and thioredoxin reductase to reduce exposed disulfides [20]. Thioredoxin cooperates with peroxiredoxins for an antiapoptotic effect through scavenging of intracellular hydrogen peroxide [21]. Serum thioredoxin levels are known to be elevated during HIV-1 infection as compared with levels in uninfected controls [22]. Elevated thioredoxin levels during HIV-1 infection demonstrate a mechanism by which cells work to control oxidative stress levels and prolong cell survival. With a decrease in thioredoxin expression during HAD, there is presumably an increase in oxidative stress, resulting in increased apoptosis and inflammatory signals.

Peroxiredoxins, thioredoxin-dependent peroxidases, protect against apoptosis by scavenging hydrogen peroxide. Peroxiredoxin 3 is expressed within the mitochondria [23]. Natural killer enhancing factors (NKEF)-A and NKEF-B, members of the peroxiredoxin family, are upregulated during HIV-1 infection in CD8+ T-cells and are thought to contribute to the antiviral activity of CD8+ T-cells [24]. Some peroxiredoxins interfere with HIV transcription by inactivation of HIV-1 long terminal repeat, thereby suppressing levels of p24 [25]. Decreases in peroxiredoxin levels of individuals with HAD may contribute to increased HIV replication and intracellular oxidative stress, later translating to neurotoxicity.

Many neurodegenerative disorders are characterized by increased oxidative stress, such as Parkinson's disease, Alzheimer's disease, and amyotrophic lateral sclerosis to name a few. Recently some oxidative stress-related proteins related to HAD have been elucidated. Hypoxia-inducible factor 1(HIF-1) is activated upon HIV-1 infection and has been found to be elevated in brains of AIDS patients [26]. The HIV-1 accessory protein, Vpr, has also been implicated in mediating HIF-1 induction [26]. In other studies, CSF and monocytes from $\mathrm{HAD}$ patients had decreased expression and activity of $\mathrm{Cu} / \mathrm{Zn}$ superoxide dismutase, indicating that oxidative stress may exacerbate HAD [19].

Traditionally, HIV-1 levels are known to be highest in the basal ganglia and hippocampus [27]. Argawal et al. investigated the loss of dopaminergic neurons in the substantia niagra due to reactive oxygen species (ROS) and GP120 administration, which was attenuated by administration of SOD or glutathione peroxidase [28]. Degeneration of the substantia nigra is a hallmark of Parkinson's disease, and therefore has been investigated in HAND due to some similarities seen between these two neurodegenerative diseases. SOD delivery in 
rodent models of GP120 neurotoxicity results in protection of dopaminergic neurons $[28,29]$. Recently, we identified SOD downregulation in monocytes of HAND-affected individuals [19]. This downregulation was confirmed in our current study in a larger number of individuals with HAND. We have also identified a down-regulation in SOD in in-vitro assays comparing viral isolates from individuals with HAD to those without neurocognitive impairment [30]. These studies prompted further investigation into changes that occur in monocyte protein expression during HAD. Downregulation of SOD combined with the loss of more specific antioxidant proteins results in unprotected ROS damage to cells.

Decreased antioxidant levels can have detrimental effects on both the affected cell and surrounding cells. Losing the ability to scavenge reactive oxygen species results in detrimental effects not just on MP, but also on astrocytes and neurons. Astrocytes are known to compensate for toxic MP secretions in in vitro models of HIV-1 infection [31], but as disease progresses this careful control of homeostasis erodes and neurotoxicity increases uncontrolled by these cells [32]. They may become overwhelmed by ROS production and therefore cannot sufficiently protect neurons in advanced stages of disease.

This study has uncovered antioxidant proteins that are downregulated in HAD. These proteins will be further investigated to understand the cause for downregulation and how to prevent or treat this deficiency. While we understand the effects of a decrease in antioxidant capabilities, what would cause such a decrease in some individuals with HIV-1 infection and not in others still remains a mystery.

\title{
Acknowledgments
}

We would like to thank our patients for supporting this research. We also wish to thank Ms. Tania for patient outreach, Drs. Rosa Hechavarría and Billy Santiago for neuropsychological testing. We thank the HIV Women Clinic (CEMI) and its Director Dr. Carmen Zorrilla and the Clinic of Sexually Transmitted Diseases (CLETS) and its Director Dr. Hermes García for referring patients to this study and the RCMI-Clinical Research Center for providing us the clinical facilities, staff, and supplies for laboratory samples. We thank Elizabeth Maldonado for patient data management. Dr. Edmundo Kraiselburd's continuous support for the project and the PR Specialized Neuroscience Program in NeuroAIDS in general are greatly appreciated.

This work was supported by NIH-NINDS grants 1U54NS430 (to L.M.M.), 2 U54 NS43011 (to V.W.), R01 MH083516-01, NIH-NCRR-RCMI-CRC-1P20RR11126, and NIH-NCRR-RCMI G12 RR-03051 for the Clinical Proteomics and Flow Cytometry core facilities (to L.M.M.), 5P01 NS31492, 2R37 NS36126, 2R01 NS034239, P20RR15635, U54NS 43011, P01 MH64570, and P01 NS43985 (to H.E.G).

\section{List of abbreviations}

\author{
HAND HIV-associated neurocognitive disorder \\ HAD HIV dmentia
}

two-dimensional image gel electrophoresis

\section{References}

1. Antinori A, Arendt G, Becker JT, Brew BJ, et al. Updated research nosology for HIV-associated neurocognitive disorders. Neurology. 2007; 69:1789-1799. [PubMed: 17914061]

2. Woods SP, Moore DJ, Weber E, Grant I. Cognitive neuropsychology of HIV-associated neurocognitive disorders. Neuropsychol Rev. 2009; 19:152-168. [PubMed: 19462243]

3. Kramer-Hammerle S, Rothenaigner I, Wolff H, Bell JE, Brack-Werner R. Cells of the central nervous system as targets and reservoirs of the human immunodeficiency virus. Virus Res. 2005; 111:194-213. [PubMed: 15885841]

4. Yadav A, Collman RG. CNS Inflammation and Macrophage/Microglial Biology Associated with HIV-1 Infection. J Neuroimmune Pharmacol. 2009 
5. Kadiu I, Glanzer JG, Kipnis J, Gendelman HE, Thomas MP. Mononuclear phagocytes in the pathogenesis of neurodegenerative diseases. Neurotox Res. 2005; 8:25-50. [PubMed: 16260384]

6. Deshpande M, Zheng J, Borgmann K, Persidsky R, et al. Role of activated astrocytes in neuronal damage: potential links to HIV-1-associated dementia. Neurotox Res. 2005; 7:183-192. [PubMed: 15897153]

7. Persidsky Y, Ghorpade A, Rasmussen J, Limoges J, et al. Microglial and astrocyte chemokines regulate monocyte migration through the blood-brain barrier in human immunodeficiency virus-1 encephalitis. Am J Pathol. 1999; 155:1599-1611. [PubMed: 10550317]

8. Dhillon NK, Williams R, Callen S, Zien C, et al. Roles of MCP-1 in development of HIV-dementia. Front Biosci. 2008; 13:3913-3918. [PubMed: 18508485]

9. Coleman CM, Wu L. HIV interactions with monocytes and dendritic cells: viral latency and reservoirs. Retrovirology. 2009; 6:51. [PubMed: 19486514]

10. Kraft-Terry SD, Buch SJ, Fox HS, Gendelman HE. A coat of many colors: neuroimmune crosstalk in human immunodeficiency virus infection. Neuron. 2009; 64:133-145. [PubMed: 19840555]

11. Rozek W, Ricardo-Dukelow M, Holloway S, Gendelman HE, et al. Cerebrospinal fluid proteomic profiling of HIV-1-infected patients with cognitive impairment. J Proteome Res. 2007; 6:4189_ 4199. [PubMed: 17929958]

12. Laspiur JP, Anderson ER, Ciborowski P, Wojna V, et al. CSF proteomic fingerprints for HIVassociated cognitive impairment. J Neuroimmunol. 2007; 192:157-170. [PubMed: 17950469]

13. Kim TA, Avraham HK, Koh YH, Jiang S, et al. HIV-1 Tat-mediated apoptosis in human brain microvascular endothelial cells. J Immunol. 2003; 170:2629-2637. [PubMed: 12594291]

14. Ciborowski P, Kadiu I, Rozek W, Smith L, et al. Investigating the human immunodeficiency virus type 1-infected monocyte-derived macrophage secretome. Virology. 2007; 363:198-209. [PubMed: 17320137]

15. Carlson KA, Ciborowski P, Schellpeper CN, Biskup TM, et al. Proteomic fingerprinting of HIV-1infected human monocyte-derived macrophages: a preliminary report. J Neuroimmunol. 2004; 147:35-42. [PubMed: 14741425]

16. Chertova E, Chertov O, Coren LV, Roser JD, et al. Proteomic and biochemical analysis of purified human immunodeficiency virus type 1 produced from infected monocyte-derived macrophages. J Virol. 2006; 80:9039-9052. [PubMed: 16940516]

17. Wojna V, Skolasky RL, Hechavarria R, Mayo R, et al. Prevalence of human immunodeficiency virus-associated cognitive impairment in a group of Hispanic women at risk for neurological impairment. J Neurovirol. 2006; 12:356-364. [PubMed: 17065128]

18. Corzett TH, Fodor IK, Choi MW, Walsworth VL, et al. Statistical analysis of the experimental variation in the proteomic characterization of human plasma by two-dimensional difference gel electrophoresis. J Proteome Res. 2006; 5:2611-2619. [PubMed: 17022632]

19. Velazquez I, Plaud M, Wojna V, Skolasky R, et al. Antioxidant enzyme dysfunction in monocytes and CSF of Hispanic women with HIV-associated cognitive impairment. J Neuroimmunol. 2009; 206:106-111. [PubMed: 19101040]

20. Holmgren A, Bjornstedt M. Thioredoxin and thioredoxin reductase. Methods Enzymol. 1995; 252:199-208. [PubMed: 7476354]

21. Fujii J, Ikeda Y. Advances in our understanding of peroxiredoxin, a multifunctional, mammalian redox protein. Redox Rep. 2002; 7:123-130. [PubMed: 12189041]

22. Nakamura H, De Rosa S, Roederer M, Anderson MT, et al. Elevation of plasma thioredoxin levels in HIV-infected individuals. Int Immunol. 1996; 8:603-611. [PubMed: 8671648]

23. Masutani H, Ueda S, Yodoi J. The thioredoxin system in retroviral infection and apoptosis. Cell Death Differ. 2005; 12(Suppl 1):991-998. [PubMed: 15818395]

24. Geiben-Lynn R, Kursar M, Brown NV, Addo MM, et al. HIV-1 antiviral activity of recombinant natural killer cell enhancing factors, NKEF-A and NKEF-B, members of the peroxiredoxin family. J Biol Chem. 2003; 278:1569-1574. [PubMed: 12421812]

25. Jin DY, Chae HZ, Rhee SG, Jeang KT. Regulatory role for a novel human thioredoxin peroxidase in NF-kappaB activation. J Biol Chem. 1997; 272:30952-30961. [PubMed: 9388242]

26. Deshmane SL, Kremlev S, Amini S, Sawaya BE. Monocyte chemoattractant protein-1 (MCP-1): an overview. J Interferon Cytokine Res. 2009; 29:313-326. [PubMed: 19441883] 
27. Wiley CA, Soontornniyomkij V, Radhakrishnan L, Masliah E, et al. Distribution of brain HIV load in AIDS. Brain Pathol. 1998; 8:277-284. [PubMed: 9546286]

28. Agrawal L, Louboutin JP, Marusich E, Reyes BA, et al. Dopaminergic neurotoxicity of HIV-1 gp120: Reactive oxygen species as signaling intermediates. Brain Res. 2009

29. Louboutin JP, Agrawal L, Reyes BA, Van Bockstaele EJ, Strayer DS. HIV-1 gp120 neurotoxicity proximally and at a distance from the point of exposure: protection by rSV40 delivery of antioxidant enzymes. Neurobiol Dis. 2009; 34:462-476. [PubMed: 19327399]

30. Toro-Nieves DM, Rodriguez Y, Plaud M, Ciborowski P, et al. Proteomic analyses of monocytederived macrophages infected with human immunodeficiency virus type 1 primary isolates from Hispanic women with and without cognitive impairment. J Neurovirol. 2009; 15:36-50. [PubMed: 19115125]

31. Wang T, Gong N, Liu J, Kadiu I, et al. Proteomic modeling for HIV-1 infected microglia-astrocyte crosstalk. PLoS ONE. 2008; 3:e2507. [PubMed: 18575609]

32. Genis P, Jett M, Bernton EW, Boyle T, et al. Cytokines and arachidonic metabolites produced during human immunodeficiency virus (HIV)-infected macrophage-astroglia interactions: implications for the neuropathogenesis of HIV disease. J Exp Med. 1992; 176:1703-1718. [PubMed: 1460427] 

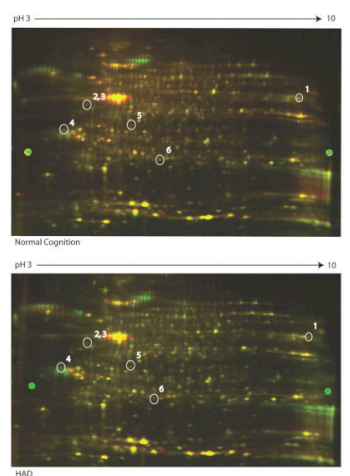

Figure 1. Representative images of 2D-DIGE differential expression and identification of proteins in CD14+ monocytes from patients with HAD by tandem mass spectrometry Differential expression of 6 protein spots of monocytes from normal cognition and HAD by 2-dimensional fluorescence imaging (2-DIGE). These were identified as 1) myeloperoxidase, 2) ADP ribosylhydrolase, 3) thioredoxin, 4) glyceraldehydes 3Pphosphate dehydrogenase; 5) Annexin I; 6) peroxiredoxin 3 


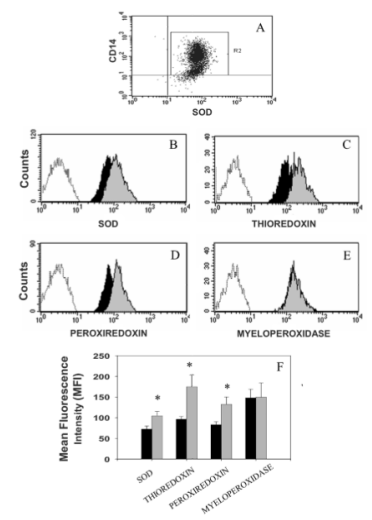

Figure 2. Intracellular expression of antioxidants in human monocytes

Monocytes gated by side scatter vs. forward scatter dot plots were identified using a CD14 monoclonal antibody (FL2 channel) and antioxidants (e.g., SOD) monoclonal antibodies (FL1 channel) (Panel A). Representative histograms of intracellular antioxidants expression show significant differences in fluorescence intensities from normal cognition (grey histogram) and HAD (dark histogram) on the expression of SOD-1 (panel B), thioredoxin (panel C), and peroxiredoxin (panel D; $\mathrm{p}<0.05$ ), but not on myeloperoxidase (panel E). A graphic representation of these differences in normal cognition (grey bars) and HAD (dark bars) is represented in panel $\mathrm{F}$. The expression of these proteins in monocytes is represented by comparison with the non-fluorescent monocytes (unshaded histograms to the left in panels B to E). 
Table 1

Demographics, viral, and immune parameters of 39 HIV-seropositive women

\begin{tabular}{llll}
\hline & Normal cognition & HAD & p value \\
\hline Age & $\mathrm{n}=20$ & $\mathrm{n}=19$ & \\
Age range & $25-51$ & $44 \pm 7$ & $\mathrm{p}=.015$ \\
CD4 & $454 \pm 214$ & $23-54$ & \\
CD4 range & $81-1062$ & $495 \pm 294$ & $\mathrm{p}=.621$ \\
CD8 & $840 \pm 316$ & $42-1258$ & \\
CD8 range & $452-1472$ & $821 \pm 555$ & $\mathrm{p}=.894$ \\
Plasma VL & $3.05 \pm 1.37$ & $38-2587$ & \\
VL range & $1.70-5.00$ & $2.24 \pm 0.93$ & $\mathrm{p}=.064$ \\
Hepatitis C & 0 & $1.70-4.48$ & \\
Therapy & & 0 & $\mathrm{p}=.704$ \\
No therapy & 2 & & $\mathrm{p}=.234$ \\
ART & 1 & 0 & \\
HAART & 14 & 15 & \\
Unknown & 3 & 0 & \\
\hline
\end{tabular}

$\mathrm{HAD}=\mathrm{HIV}$-associated dementia 


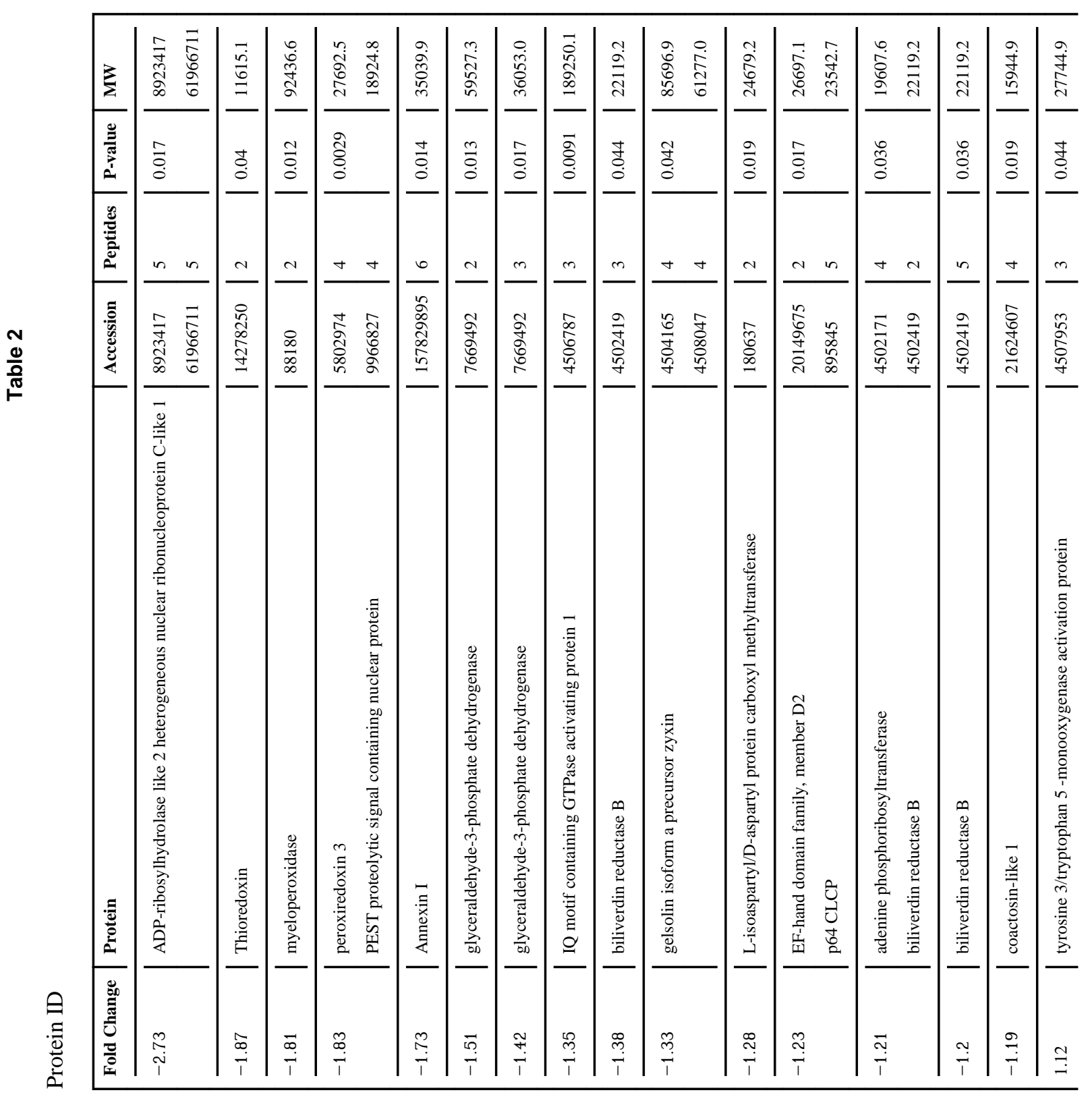




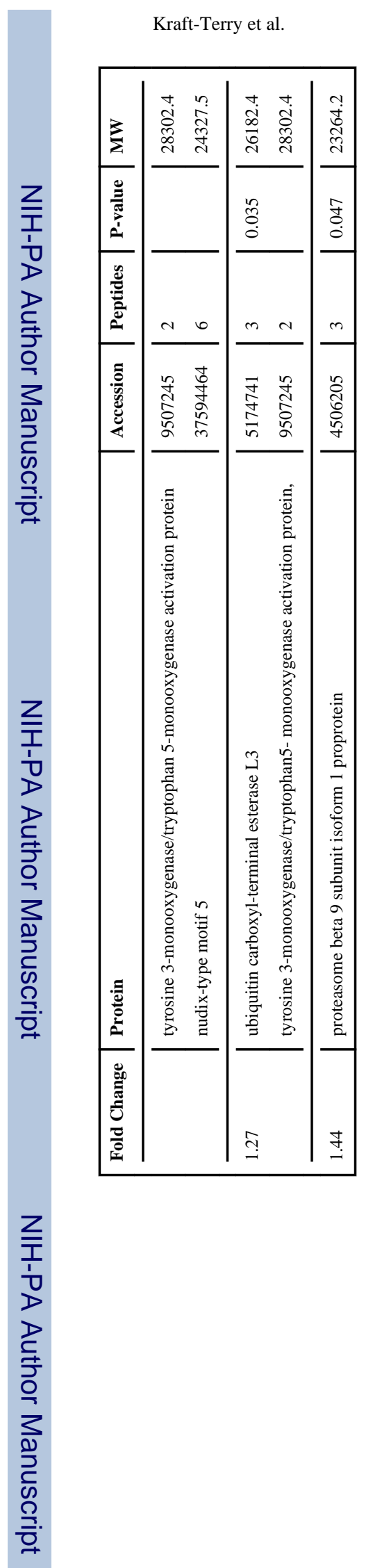

Proteomics Clin Appl. Author manuscript; available in PMC 2011 May 20. 